\title{
STRONG SYSTEM EQUIVALENCE (II)
}

\author{
W. A. COPPEL ${ }^{1}$ AND D. J. CULLEN ${ }^{1}$
}

(Received 17 August 1984; revised 28 November 1984)

\begin{abstract}
The notion of strong system equivalence, which was defined and studied in Anderson, Coppel and Cullen [1], is here given a module-theoretic characterization and a dynamical interpretation.
\end{abstract}

\section{Introduction}

The present work is intended to elucidate further the notions of system equivalence at infinity and strong system equivalence, which were defined and studied in [1]. It will be shown that they can be given module-theoretic characterizations completely analogous to the module-theoretic characterization of ordinary system equivalence. An interpretation in terms of differential equations will also be given.

Let $K$ be an arbitrary field and let $K(s)$ be the field of rational functions with coefficients from $K$. Any rational function $r(s)$ in $K(s)$ can be uniquely expressed in the form

$$
r(s)=q(s)+\sigma(s)
$$

where $q(s)$ is a polynomial and $\sigma(s)$ is a strictly causal rational function. If we write $\sigma(s)=\pi r(s)$ then the linear operator $\pi$ is a projection: $\pi^{2}=\pi$. Moreover

$$
\pi p \pi r=\pi p r
$$

for any rational function $r$ and any polynomial $p$, since $\pi p(I-\pi) r=0$. Similarly

$$
(I-\pi) \rho(I-\pi) r=(I-\pi) \rho r
$$

\footnotetext{
${ }^{1}$ Department of Mathematics, Research School of Physical Sciences, Australian National University, G.P.O. Box 4, Canberra, A.C.T. 2601, Australia.

(1) Copyright Australian Mathematical Society 1985, Serial-fee code 0334-2700/85
} 
for any rational function $r$ and any causal rational function $\rho$. These definitions and relations extend at once from scalar to matrix functions.

The following two lemmas may be known, but we were unable to provide a reference for them in the form we require. They will play no direct role in our work, but serve to interpret it in Section 4 .

Consider the homogeneous system of linear differential equations

$$
T(D) \xi=0,
$$

where $D=d / d t$ and $T(s)$ is a non-singular $n \times n$ matrix of polynomials with constant (complex) coefficients. We wish to characterize the solutions of (1) in the frequency domain.

LEMMA 1. The Laplace transforms of the solutions $\xi(t)$ of (1) are precisely those strictly causal rational functions $x(s)$ for which $T(s) x(s)$ is a polynomial vector.

They form a (complex) vector space of dimension $=\operatorname{deg} \operatorname{det} T(s)$.

Proof. There exists a bipolynomial matrix $U(s)$ such that $\bar{T}(s)=T(s) U(s)$ is in Hermite canonical form. That is,

$$
\bar{t}_{j k}=0 \quad \text { if } j<k, \quad \bar{t}_{j j} \text { is monic, } \delta\left(\bar{t}_{j k}\right)<\delta\left(\bar{t}_{j j}\right) \text { if } j>k .
$$

Moreover $\bar{T}(s)$ is uniquely determined by these requirements, and hence $U(s)$ is also.

If $\bar{x}(s)$ is the Laplace transform of a solution $\bar{\xi}(t)$ of the transformed system of differential equations

$$
\bar{T}(D) \bar{\xi}(t)=0,
$$

then

$$
\begin{aligned}
& \bar{t}_{11}(s) \bar{x}_{1}(s)=p_{1}(s) \\
& \bar{t}_{21}(s) \bar{x}_{1}(s)+\bar{t}_{22}(s) \bar{x}_{2}(s)=p_{2}(s) \ldots \ldots \ldots \ldots \ldots \ldots \ldots \ldots \ldots \ldots \ldots \ldots \ldots \ldots \ldots \ldots \\
& \cdots \cdots \\
& \bar{t}_{n 1}(s) \bar{x}_{1}(s)+\bar{t}_{n 2}(s) \bar{x}_{2}(s)+\cdots \cdots+\bar{t}_{n n}(s) \bar{x}_{n}(s)=p_{n}(s)
\end{aligned}
$$

where $p_{j}(s)$ is an arbitrary polynomial of lower degree than $\bar{t}_{j j}(s)(j=1, \ldots, n)$, which is determined by the initial conditions. Conversely, any such $\bar{x}(s)$ is the Laplace transform of a solution $\bar{\xi}(t)$ of (2). It follows that Lemma 1 holds for the system (2). Thus the solutions $\bar{\xi}(t)$ of (2) are exponential polynomials.

The solutions of (1) are given by $\xi(t)=U(D) \bar{\xi}(t)$, where $\bar{\xi}(t)$ is an arbitrary solution of (2), and thus are also exponential polynomials. Hence the Laplace transform $x(s)$ of the solution $\xi(t)$ of (1) is a strictly causal rational function. 
Moreover, $x(s)=U(s) \bar{x}(s)+q(s)$, where $q(s)$ is a polynomial vector determined by the initial conditions, and hence

$$
T(s) x(s)=\bar{T}(s) \bar{x}(s)+T(s) q(s)
$$

is a polynomial vector.

Conversely, if $x(s)$ is a strictly causal rational function such that $T(s) x(s)$ is a polynomial vector then $x(s)$ is the Laplace transform of an exponential polynomial $\xi(t)$. Hence $\bar{\xi}=U^{-1}(D) \xi(t)$ is also an exponential polynomial and its Laplace transform $\bar{x}(s)$ is a strictly causal rational function. Since

$$
\bar{x}(s)=U^{-1}(s) x(s)+\bar{q}(s),
$$

where $\bar{q}(s)$ is a polynomial vector,

$$
\bar{T}(s) \bar{x}(s)=T(s) x(s)+\bar{T}(s) \bar{q}(s)
$$

is also a polynomial vector. Consequently $\bar{\xi}(t)$ is a solution of (2) and $\xi(t)=$ $U(D) \bar{\xi}(t)$ is a solution of (1). In this way the validity of Lemma 1 for (2) implies its validity also for (1).

Since we are interested in the behaviour of linear systems at infinity we must consider also the (pure) impulsive, or distributional, solutions of (1). An examination of the discussion in Verghese [4, pages 93-95] yields the following counterpart to Lemma 1.

LEMMA 2. The Laplace transforms of the impulsive solutions $\xi_{\infty}(t)$ of (1) are precisely those polynomial functions $x_{\infty}(s)$ which are the polynomial parts of rational vectors $r(s)$ for which $T(s) r(s)$ is strictly causal.

They form a vector space of dimension $=\operatorname{deg} \operatorname{det} T_{-}^{-1}$, where in the notation of $[1]$, $\left[T_{+}, T_{0}, T_{-}\right]$is the Smith-McMillan form at infinity of $T$.

It follows that all ordinary and impulsive solutions of (1) form a vector space of dimension equal to the McMillan degree $\delta(T)$, since $\delta(\operatorname{det} T)-\delta\left(\operatorname{det} T_{-}\right)=$ $\delta\left(\operatorname{det} T_{+}\right)=\delta(T)$. In the notation introduced at the beginning of this section, the set of Laplace transforms of ordinary solutions of (1) is $\pi T^{-1}(s)(I-\pi) \mathrm{C}^{n}(s)$, and the set of Laplace transforms of impulsive solutions of equation (1) is $(I-\pi) T^{-1}(s) \pi \mathbf{C}^{n}(s)$.

\section{System equivalence at infinity}

Let

$$
R=W+V T^{-1} U
$$

be a polynomial realization of the $p \times m$ rational matrix $R$. We recall from [1] 
that with this realization there is associated the extended system matrix

$$
Q=\left[\begin{array}{ccc}
-T & U & 0 \\
V & W & -I_{p} \\
0 & I_{m} & 0
\end{array}\right]
$$

Moreover

$$
P=\mathscr{Q} \mathscr{Q}^{-1} \mathscr{R}
$$

where

$$
\mathscr{B}=\left[\begin{array}{c}
0 \\
0 \\
I_{m}
\end{array}\right], \quad \mathscr{C}=\left[\begin{array}{lll}
0 & 0 & I_{p}
\end{array}\right],
$$

and the realizations (3) and (4) are strongly system equivalent. These definitions of $Q, \mathscr{B}$, and $\mathscr{C}$ will be retained throughout the present paper.

We show first that Proposition 1 of [1] can be stated in an apparently stronger form.

\section{Proposition 1. Two polynomial realizations}

$$
R=W+V T^{-1} U=\tilde{W}+\tilde{V} \tilde{T}^{-1} \tilde{U}
$$

of a rational matrix $R$, with extended system matrices $Q$ and $\tilde{Q}$, are system equivalent at infinity if and only if there exist causal matrices $\mathscr{M}, \mathscr{N}, \mathscr{X}, \mathscr{Y}$ such that $[\mathscr{M} \tilde{Q}]$ has a causal right inverse, $\left[{ }_{Q}^{\mathcal{L}}\right]$ has a causal left inverse, and

$$
\left[\begin{array}{cc}
\mathscr{M} & 0 \\
\mathscr{X} & I
\end{array}\right]\left[\begin{array}{cc}
-Q & \mathscr{B} \\
\mathscr{C} & 0
\end{array}\right]=\left[\begin{array}{cc}
-\tilde{Q} & \tilde{\mathscr{B}} \\
\tilde{\mathscr{C}} & 0
\end{array}\right]\left[\begin{array}{cc}
\mathscr{N} & \mathscr{Y} \\
0 & I
\end{array}\right]
$$

Proof. By Proposition 1 of [1] it is sufficient to show that if there exist causal matrices $\mathscr{M}, \mathscr{N}, \mathscr{X}, \mathscr{Y}$ with these properties then there exist causal matrices $\mathscr{M}, \mathscr{N}$, $\mathscr{X}, \mathscr{Y}$ with the same properties and such that, in addition, the common value of both sides of (6) is causal.

Let

$$
Q^{-1}=\mathscr{T}^{-1} \mathscr{U}, \quad \tilde{Q}^{-1}=\tilde{\mathscr{V}} \tilde{T}^{-1}
$$

be irreducible causal realizations. By hypothesis there exist causal matrices $\mathscr{F}, \mathscr{G}$ such that

$$
\mathscr{M} \mathscr{F}+\tilde{Q} \mathscr{G}=I
$$

Thus $\tilde{Q} \mathscr{G}$ is causal, and hence $\mathscr{G}=\tilde{\mathscr{V}} \overline{\mathscr{G}}$ for some causal matrix $\overline{\mathscr{G}}$. It follows that $\mathscr{M}$ and $\mathscr{T}$ are left coprime. Similarly $\mathscr{N}$ and $\mathscr{T}$ are right coprime. But $\mathscr{M} Q=\tilde{Q} \mathscr{N}$ implies

$$
\tilde{\mathscr{V}} \tilde{\mathscr{T}}^{-1} \mathscr{M}=\mathscr{N} \mathscr{T}^{-1} \mathscr{U}
$$


Moreover both these realizations are irreducible, and consequently they are system equivalent. Thus there exist causal matrices $\mathscr{K}, \mathscr{L}, \mathscr{W}, \mathscr{Z}$ such that

$$
\left[\begin{array}{cc}
\mathscr{K} & 0 \\
\mathscr{W} & I
\end{array}\right]\left[\begin{array}{cc}
-\mathscr{T} & \mathscr{U} \\
\mathscr{N} & 0
\end{array}\right]=\left[\begin{array}{cc}
-\tilde{T} & \mathscr{M} \\
\tilde{\mathscr{V}} & 0
\end{array}\right]\left[\begin{array}{cc}
\mathscr{L} & \mathscr{Z} \\
0 & I
\end{array}\right]
$$

In particular

$$
\mathscr{W} \mathscr{U}=\tilde{\mathscr{Y}} \mathscr{Z}=\mathscr{H}, \text { say, }
$$

and

$$
\mathscr{N}-\mathscr{W} \mathscr{T}=\tilde{\mathscr{V}} \mathscr{L}
$$

Put

$$
\begin{array}{ll}
\overline{\mathscr{M}}=\mathscr{M}-\tilde{Q} \mathscr{H}, & \overline{\mathcal{N}}=\mathscr{N}-\mathscr{H} Q, \\
\overline{\mathscr{X}}=\mathscr{X}+\tilde{\mathscr{C}} \mathscr{H}, & \overline{\mathscr{Y}}=\mathscr{Y}+\mathscr{H} \mathscr{B} .
\end{array}
$$

Then $\overline{\mathscr{M}}, \overline{\mathcal{N}}, \overline{\mathscr{X}}, \overline{\mathscr{Y}}$ are causal and

$$
\left[\begin{array}{cc}
\overline{\mathscr{M}} & 0 \\
\overline{\mathscr{X}} & I
\end{array}\right]\left[\begin{array}{cc}
-Q & \mathscr{B} \\
\mathscr{C} & 0
\end{array}\right]=\left[\begin{array}{cc}
-\tilde{Q} & \tilde{\mathscr{B}} \\
\tilde{\mathscr{C}} & 0
\end{array}\right]\left[\begin{array}{cc}
\overline{\mathscr{N}} & \overline{\mathscr{Y}} \\
0 & I
\end{array}\right]
$$

where the common value of both sides is causal. Moreover $[\overline{\mathscr{M}} \tilde{Q}]$ has a causal right inverse, since

$$
\overline{\mathscr{M}} \mathscr{F}+\tilde{Q}(\mathscr{G}+\mathscr{H} \mathscr{F})=I,
$$

and similarly $\left[\begin{array}{l}\bar{T} \\ Q\end{array}\right]$ has a causal left inverse.

Proposition 2 of [1] can also be stated in an apparently stronger form: the realizations (5) are system equivalent at infinity if and only if there exist causal matrices $\mathscr{M}, \mathscr{N}, \mathscr{X}, \mathscr{Y}$ such that

$$
\left[\begin{array}{cc}
\mathscr{M} & 0 \\
\mathscr{X} & I
\end{array}\right]\left[\begin{array}{ccc}
I & 0 & 0 \\
0 & -\mathscr{Q} & \mathscr{B} \\
0 & \mathscr{C} & 0
\end{array}\right]=\left[\begin{array}{ccc}
I & 0 & 0 \\
0 & -\tilde{Q} & \tilde{\mathscr{B}} \\
0 & \tilde{\mathscr{C}} & 0
\end{array}\right]\left[\begin{array}{cc}
\mathscr{N} & \mathscr{Y} \\
0 & I
\end{array}\right],
$$

ii) $\quad \mathscr{G}\left[\begin{array}{cc}I & 0 \\ 0 & -Q\end{array}\right] \quad \begin{aligned} & \text { is causal for some causal } \mathscr{G} \text { if and only if } \\ & \mathscr{G}=\overline{\mathscr{G}} \mathscr{M} \text { for some causal } \overline{\mathscr{G}} \text {, }\end{aligned}$

(iii) $\quad\left[\begin{array}{cc}I & 0 \\ 0 & -\tilde{Q}\end{array}\right] \mathscr{H} \begin{aligned} & \text { is causal for some causal } \mathscr{H} \text { if and only if } \\ & \mathscr{H}=\mathscr{N} \overline{\mathscr{H}} \text { for some causal } \overline{\mathscr{H}} \text {. }\end{aligned}$

The strengthening lies in the fact that $\mathscr{X}$ and $\mathscr{Y}$ in (i) are not required to be divisible by $\mathscr{M}$ and $\mathscr{N}$. The result follows without difficulty from the preceding Proposition 1, and the proof is omitted.

Again let $R$ be a $p \times m$ rational matrix with the polynomial realization (3). If the corresponding extended system matrix $Q$ is of size $l \times l$, the vector space

$$
\mathscr{X}_{\infty}=(I-\pi) Q^{-1} \pi K^{\prime}(s)
$$


will be called the state-space at infinity of the realization (3). For any $x \in \mathscr{X}_{\infty}$ and any causal rational function $\rho$ set

$$
\rho \cdot x=(I-\pi) \rho x .
$$

Then $\mathscr{X}_{\infty}$ becomes a module over the ring of causal functions, since the product is obviously linear in both factors and for any causal $\sigma$

$$
\begin{aligned}
\sigma \cdot(\rho \cdot x) & =(I-\pi) \sigma(I-\pi) \rho x \\
& =(I-\pi) \sigma \rho x \\
& =(\sigma \rho) \cdot x .
\end{aligned}
$$

Suppose now that we have two polynomial realizations (5) of the rational matrix $R$. Let $Q, \tilde{Q}$ be the corresponding extended system matrices and $\mathscr{X}_{\infty}, \tilde{\mathscr{X}}_{\infty}$ the corresponding state-spaces at infinity. We are going to obtain a characterization of all module homomorphisms from $\mathscr{X}_{\infty}$ into $\tilde{\mathscr{X}}_{\infty}$.

Proposition 2. For any causal matrix $\mathcal{N}$ such that $\mathscr{M}=\tilde{Q} \mathscr{N} Q^{-1}$ is causal, the map

$$
f_{\infty}: x \rightarrow(I-\pi) \mathcal{N} x
$$

is a homomorphism of the causal module $\mathscr{X}_{\infty}$ into the causal module $\tilde{\mathscr{X}}_{\infty}$. Moreover any homomorphism $f_{\infty}: \mathscr{X}_{\infty} \rightarrow \tilde{X}_{\infty}$ can be defined in this way.

The map $f_{\infty}: \mathscr{X}_{\infty} \rightarrow \tilde{\mathscr{X}}_{\infty}$ is surjective if and only if $[\mathscr{M} \tilde{Q}]$ has a causal right inverse, and injective if and only if $\left[{ }_{Q}^{\mathcal{H}}\right]$ has a causal left inverse.

Proof. If $x=(I-\pi) Q^{-1} \rho$, where $\rho$ is strictly causal, then

$$
f_{\infty}(x)=(I-\pi) \mathcal{N} Q^{-1} \rho=(I-\pi) \tilde{Q}^{-1} \tilde{\rho},
$$

where $\tilde{\rho}=\mathscr{M} \rho$ is also strictly causal. Thus $f_{\infty}$ maps $\mathscr{X}_{\infty}$ into $\tilde{\mathscr{X}}_{\infty}$. Moreover it is a homomorphism, since for any causal $\sigma$

$$
\begin{aligned}
f_{\infty}(\sigma \cdot x) & =(I-\pi) \mathscr{N}(I-\pi) \sigma x \\
& =(I-\pi) \mathscr{N} \sigma x \\
& =(I-\pi) \sigma(I-\pi) \mathscr{N} x \\
& =\sigma \cdot f_{\infty}(x) .
\end{aligned}
$$

Conversely, let $f_{\infty}$ be any homomorphism of $\mathscr{X}_{\infty}$ into $\tilde{\mathscr{X}}_{\infty}$. If $e_{1}, \ldots, e_{l}$ are the columns of the $l \times l$ unit matrix, then

$$
f_{\infty}\left((I-\pi) s^{-1} Q^{-1} e_{k}\right)=(I-\pi) s^{-1} \tilde{Q}^{-1} \tilde{n}_{k}
$$

for some causal vector $\tilde{n}_{k}(k=1, \ldots, l)$. Let $\tilde{N}$ be the $\tilde{l} \times l$ causal matrix with columns $\tilde{n}_{1}, \ldots, \tilde{n}_{l}$. then, since $f_{\infty}$ is a homomorphism,

$$
f_{\infty}\left((I-\pi) Q^{-1} \rho\right)=(I-\pi) \tilde{Q}^{-1} \tilde{\mathcal{N}} \rho
$$

for any strictly causal vector $\rho$. 
Let

$$
Q^{-1}=\mathscr{V} \mathscr{T}^{-1}, \quad \tilde{Q}^{-1}=\tilde{\mathscr{V}} \tilde{\mathscr{T}}^{-1}
$$

be irreducible causal realizations. Since the homomorphism $f_{\infty}$ maps zero into zero, by taking $\rho=s^{-1} \mathscr{T}$ in (8) we see that $\tilde{Q}^{-1} \tilde{\mathcal{N}} \mathscr{T}$ is causal. Hence

$$
\tilde{\mathscr{N}} \mathscr{T}=\tilde{\mathscr{T}} \overline{\mathscr{H}}
$$

for some causal $\overline{\mathscr{H}}$. Since $\mathscr{T}$ and $\mathscr{V}$ are right coprime, there exist causal $\overline{\mathscr{F}}, \overline{\mathscr{G}}$ such that

$$
\overline{\mathscr{F}} \mathscr{T}+\overline{\mathscr{G}} \mathscr{V}=\overline{\mathscr{H}}
$$

If we put $\mathscr{M}=\tilde{\mathscr{N}}-\tilde{\mathscr{T}} \overline{\mathscr{F}}$ then $\mathscr{N}=\tilde{Q}^{-1} \mathscr{M} Q$ is causal, since

$$
\begin{aligned}
\mathscr{N} & =\tilde{Q}^{-1} \tilde{\mathscr{N}} Q-\tilde{\mathscr{V}} \overline{\mathscr{F}} Q \\
& =\tilde{Q}^{-1} \tilde{\mathscr{T}} \overline{\mathscr{H}} \mathscr{V}^{-1}-\tilde{\mathscr{V}} \overline{\mathscr{F}} \mathscr{T} \mathscr{V}^{-1} \\
& =\tilde{\mathscr{V}}(\overline{\mathscr{H}}-\overline{\mathscr{F}} \mathscr{T}) \mathscr{V}^{-1} \\
& =\tilde{\mathscr{V}} \overline{\mathscr{G}} .
\end{aligned}
$$

On the other hand, since $\tilde{Q}^{-1} \tilde{\mathscr{T}} \overline{\mathscr{F}}$ is causal, it follows from (8) that for any strictly causal vector $\rho$

$$
\begin{aligned}
f_{\infty}\left((I-\pi) Q^{-1} \rho\right) & =(I-\pi) \tilde{Q}^{-1} \mathscr{M} \rho \\
& =(I-\pi) \mathscr{N} Q^{-1} \rho .
\end{aligned}
$$

Hence for any $x \in \mathscr{X}_{\infty}$.

$$
f_{\infty}(x)=(I-\pi) \mathscr{N} x .
$$

Suppose there exist causal $\mathscr{F}, \mathscr{G}$ such that

$$
\mathscr{M} \mathscr{F}+\tilde{Q} \mathscr{G}=I \text {. }
$$

If $\tilde{x}=(I-\pi) \tilde{Q}^{-1} \tilde{\rho}$, where $\tilde{\rho}$ is strictly causal, then

$$
\begin{aligned}
\tilde{x} & =(I-\pi)\left(\mathscr{N} Q^{-1 \mathscr{F}+\mathscr{G}) \tilde{\boldsymbol{\rho}}}\right. \\
& =(I-\pi) \mathscr{N} Q^{-1 \mathscr{F} \tilde{\boldsymbol{\rho}}} \\
& =(I-\pi) \mathscr{N} x,
\end{aligned}
$$

where $x=(I-\pi) Q^{-1 \mathscr{F}} \tilde{\rho}$. Thus the map $f_{\infty}: \mathscr{X}_{\infty} \rightarrow \tilde{X}_{\infty}$ is surjective.

Conversely, if the map $f_{\infty}$ is surjective there exists a causal matrix $\mathscr{F}$ such that

$$
(I-\pi)\left(s^{-1} \tilde{Q}^{-1}\right)=(I-\pi)\left(\mathscr{N} Q^{-1} s^{-1} \mathscr{F}\right) .
$$

That is, $\tilde{Q}^{-1}=\mathscr{N} Q^{-1} \mathscr{F}+\mathscr{G}$ for some causal $\mathscr{G}$. Hence $[\mathscr{M} \tilde{Q}]$ has a causal right inverse.

Suppose there exist causal $\tilde{F}, \tilde{\mathscr{G}}$ such that

$$
\tilde{\mathscr{F}} \mathscr{N}+\tilde{\mathscr{G}} Q=1
$$

If $(I-\pi) \mathscr{N} x=0$, where $x=(I-\pi) Q^{-1} \rho$ for some strictly causal $\rho$, then $(I-\pi) \mathscr{N} Q^{-1} \rho=0$. Thus $\mathcal{N} Q^{-1} \rho$ is strictly causal. Since $\tilde{\mathscr{G}} \rho$ is strictly causal, it follows that $Q^{-1} \rho$ is strictly causal and hence $x=0$. Thus the map $f_{\infty}$ is injective. 
Conversely, if the map $f_{\infty}$ is injective then $\mathscr{N} Q^{-1} \rho$ strictly causal for some strictly causal $\rho$ implies that $Q^{-1} \rho$ is strictly causal. Let

$$
\mathscr{N} Q^{-1}=\mathscr{H} \mathscr{K}^{-1}
$$

be an irreducible causal realization. Then if we take $\rho=s^{-1} \mathscr{K}$ it follows that $\mathscr{L}=Q^{-1} \mathscr{K}$ is causal. Since $\mathscr{H}$ and $\mathscr{K}$ are right coprime, there exist causal $\tilde{\mathscr{F}}, \tilde{\mathscr{G}}$ such that

Then

$$
\tilde{\mathscr{F}} \mathscr{H}+\tilde{\mathscr{G}} \mathscr{K}=\mathscr{L}
$$

$$
Q^{-1}=\tilde{\mathscr{F}} \mathcal{N} Q^{-1}+\tilde{\mathscr{G}}
$$

and hence $[\underset{Q}{\mathcal{N}}]$ has a causal left inverse. This completes the proof.

We can now obtain without difficulty a module-theoretic characterization of system equivalence at infinity.

Proposition 3. Let

$$
R=W+V T^{-1} U=\tilde{W}+\tilde{V} \tilde{T}^{-1} \tilde{U}
$$

be polynomial realizations of the $p \times m$ rational matrix $R$. Let $Q, \tilde{Q}$ be the corresponding extended system matrices and $\mathscr{X}_{\infty}, \tilde{\mathscr{X}}_{\infty}$ the corresponding state-spaces at infinity.

Then the realizations (5) are system equivalent at infinity if and only there exists a module isomorphism $f_{\infty}: \mathscr{X}_{\infty} \rightarrow \tilde{\mathscr{X}}_{\infty}$ such that the following diagram commutes.

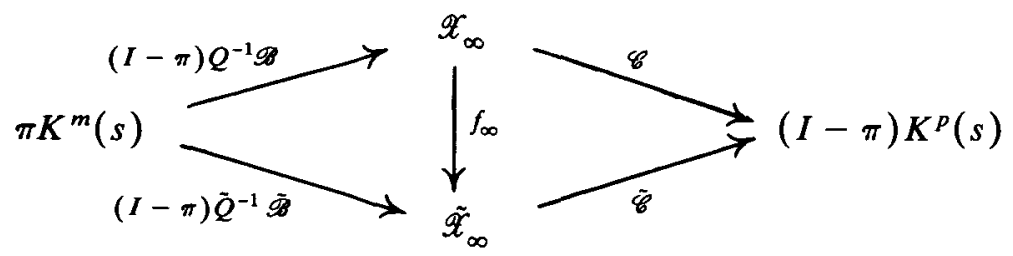

Figure 1. System-equivalence at infinity.

Proof. Suppose first that the realizations (5) are system equivalent at infinity. Then there exist causal matrices $\mathscr{M}, \mathscr{N}, \mathscr{X}, \mathscr{Y}$ such that $[\mathscr{M} \tilde{Q}]$ has a causal right inverse, $\left[{ }_{Q}^{\mathscr{N}}\right]$ has a causal left inverse, and

$$
\left[\begin{array}{cc}
\mathscr{K} & 0 \\
\mathscr{X} & I
\end{array}\right]\left[\begin{array}{cc}
-Q & \mathscr{B} \\
\mathscr{C} & 0
\end{array}\right]=\left[\begin{array}{cc}
-\tilde{Q} & \tilde{\mathscr{B}} \\
\tilde{\mathscr{C}} & 0
\end{array}\right]\left[\begin{array}{cc}
\mathscr{N} & \mathscr{Y} \\
0 & I
\end{array}\right]
$$


Since $\mathscr{N}$ and $\mathscr{M}=\tilde{Q} \mathscr{N} Q^{-1}$ are causal, the map $f_{\infty}(x)=(I-\pi) \mathscr{N} x$ defines an isomorphism of $\mathscr{X}_{\infty}$ with $\tilde{\mathscr{X}}_{\infty}$, by Proposition 2. Moreover if $x=(I-\pi) Q^{-1} \rho$, where $\rho$ is strictly causal, then

$$
\begin{aligned}
\tilde{\mathscr{C}}_{\infty}(x) & =(I-\pi)\left(\tilde{\mathscr{C}} \mathscr{N} Q^{-1} \rho\right) \\
& =(I-\pi)\left(\mathscr{C} Q^{-1} \rho-\mathscr{X} \rho\right) \\
& =\mathscr{C} x,
\end{aligned}
$$

since $\tilde{\mathscr{C}}$ is constant and $\mathscr{X} \rho$ strictly causal. Thus the right half of the diagram commutes.

Again, for any strictly causal $\rho$, if $x=(I-\pi) Q^{-1} \mathscr{B} \rho$ then

$$
\begin{aligned}
f_{\infty}(x) & =(I-\pi)\left(\mathscr{N} Q^{-1} \mathscr{B} \rho\right) \\
& =(I-\pi)\left(\tilde{Q}^{-1} \tilde{\mathscr{B}} \rho-\mathscr{Y} \rho\right) \\
& =(I-\pi) \tilde{Q}^{-1} \tilde{\mathscr{B}} \rho
\end{aligned}
$$

since $\mathscr{Y} \rho$ is strictly causal. Thus the left half of the diagram commutes.

Conversely, suppose there exists an isomorphism $f_{\infty}$ which makes the diagram in Figure 1 commute. If $\mathscr{N}$ and $\mathscr{M}=\tilde{Q} \mathscr{N} Q^{-1}$ are the causal matrices associated with $f_{\infty}$ by Proposition 2, then [ $\mathscr{M} \tilde{Q}$ ] has a causal right inverse and $\left[\left[_{Q}^{\mathscr{H}}\right.\right.$ ] has a causal left inverse. The previous argument that the right and left halves of the diagram commute can be reversed to show that the matrices

$$
\mathscr{X}=\mathscr{C} Q^{-1}-\tilde{\mathscr{C}} \mathscr{N} Q^{-1}
$$

and

$$
\mathscr{Y}=\tilde{Q}^{-1} \tilde{\mathscr{B}}-\mathscr{N} Q^{-1} \mathscr{B}
$$

are causal. Since

$$
R=\mathscr{C} Q^{-1} \mathscr{B}=\tilde{\mathscr{C}} \tilde{Q}^{-1} \tilde{\mathscr{B}},
$$

if follows that (6) holds. Thus the realizations (5) are system equivalent at infinity.

Controllability and observability at infinity can also be interpreted in terms of the preceding diagram.

Proposition 4. Let $R$ be a $p \times m$ rational matrix with the polynomial realization

$$
R=W+V T^{-1} U
$$

and let $Q$ be the corresponding extended system matrix. Then the realization (3) is controllable at infinity if and only if the map

$$
(I-\pi) Q^{-1} \mathscr{B}: \pi K^{m}(s) \rightarrow \mathscr{X}_{\infty}
$$

is surjective, and it is observable at infinity if and only if the map

$$
\mathscr{C}: \mathscr{X}_{\infty} \rightarrow(I-\pi) K^{p}(s)
$$

is injective. 
Proof. The given realization is strongly system equivalent to the realization $R=\mathscr{C} Q^{-1} \mathscr{B}$. Consequently, by Proposition 4 of [1], it is controllable at infinity if and only if the matrix

$$
\left[\begin{array}{ccc}
-Q & \mathscr{B} & 0 \\
\mathscr{C} & 0 & -I
\end{array}\right]
$$

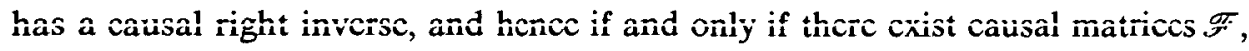
$\mathscr{G}$ such that

$$
Q \mathscr{F}+\mathscr{B} \mathscr{G}=I .
$$

If this is the case then, for any strictly causal $\rho$,

$$
(I-\pi)\left(Q^{-1} \rho\right)=(I-\pi)\left(Q^{-1} \mathscr{B} \mathscr{G} \rho\right) .
$$

Thus the map $Q^{-1 \mathscr{B}:} \pi K^{m}(s) \rightarrow \mathscr{X}_{\infty}$ is surjective. This argument can be reversed.

Similarly the realization (3) is observable at infinity if and only if there exist causal matrices $\tilde{\mathscr{F}}, \tilde{\mathscr{G}}$ such that

$$
\tilde{\mathscr{F}} Q+\tilde{\mathscr{G}} \mathscr{C}=I \text {. }
$$

If this is the case then by multiplying on the right by $Q^{-1} \rho$, we see that $(I-\pi) \mathscr{C} Q^{-1} \rho=0$ for some strictly causal $\rho$ implies $(I-\pi) Q^{-1} \rho=0$. Thus the map $\mathscr{C}: \mathscr{X}_{\infty} \rightarrow(I-\pi) K^{p}(s)$ is injective. Again the argument can be reversed.

\section{Strong system equivalence}

The characterization of system equivalence at infinity given by Proposition 3 is completely analogous to the module-theoretic characterization of ordinary system equivalence given in [3] and [2]. In fact let $R$ be a $p \times m$ rational matrix with the polynomial realization (3), and let $Q$ be the corresponding extended system matrix. Since the realization (3) is strongly system equivalent to the realization

$$
R=\mathscr{C} Q^{-1} \mathscr{B},
$$

we can express everything in terms of the latter. The vector space

$$
\mathscr{X}=\pi Q^{-1}(I-\pi) K^{\prime}(s)
$$

is the state-space of the realization. Moreover, $\mathscr{X}$ becomes a module over the ring of polynomials if, for any $x \in \mathscr{X}$ and any polynomial $p$, we define

$$
p \cdot x=\pi(p x) \text {. }
$$


The realizations (5) are system equivalent if and only if there exists a module isomorphism $f: \mathscr{X} \rightarrow \tilde{X}$ such that the following diagram commutes:

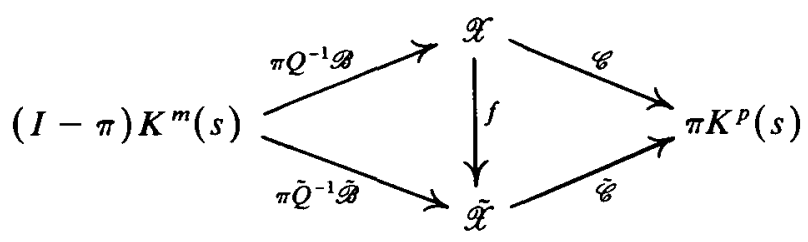

Figure 2. System equivalence.

Furthermore, the realization (3) is controllable if and only if the map

$$
\pi Q^{-1 \mathscr{B}:}(I-\pi) K^{m}(s) \rightarrow \mathscr{X}
$$

is surjective, and it is observable if and only if the map

$$
\mathscr{C}: \mathscr{X} \rightarrow \pi K^{p}(s)
$$

is injective. It will be observed that the results for behaviour at infinity are obtained by simply replacing the state space by the state space at infinity and interchanging $\pi$ and $I-\pi$.

These results can be combined to give a module-theoretic characterization of strong system equivalence. For any rational matrix $S$ and rational vector $r$ let $\llbracket S \rrbracket$ denote the linear map

$$
\llbracket S \rrbracket: r \rightarrow \pi S(I-\pi) r+(I-\pi) S \pi r .
$$

The $K$-vector space

$$
\mathscr{X}_{s}=\llbracket Q^{-1} \rrbracket K^{l}(s)
$$

will be called the strong state-space of the realization (3). It is the (vector space) direct sum of the state-space and the state-space at infinity. The realizations (5) are strongly system equivalent if and only if there exists a linear map $f_{s}: \mathscr{X}_{s} \rightarrow \tilde{\mathscr{X}}_{s}$ which maps $\mathscr{X}$ isomorphically onto $\tilde{\mathscr{X}}$, and $\mathscr{X}_{\infty}$ isomorphically onto $\tilde{\mathscr{X}}_{\infty}$, and which makes the following diagram commute:

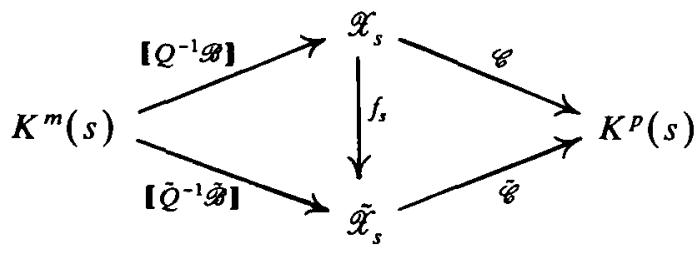

Figure 3. Strong system equivalence. 
The realization (3) is strongly controllable if and only if the map

$$
\llbracket Q^{-1} \mathscr{B} \rrbracket: K^{m}(s) \rightarrow \mathscr{X}_{s}
$$

is surjective, and strongly observable if and only if the map

$$
\mathscr{C}: \mathscr{X}_{s} \rightarrow K^{p}(s)
$$

is injective.

More conceptual proofs of Theorems 1 and 2 of [1] can be based on these results.

\section{Dynamical interpretation}

In this section we consider the interpretation of the preceding results in the time domain, when the underlying field $K$ is the field $\mathbf{C}$ of complex numbers. Since this is simply a matter of taking inverse Laplace transforms, we will usually just state the result.

We are now given a polynomial system of differential equations

$$
T(D) x=U(D) u, \quad y=V(D) x+W(D) u,
$$

and we are interested in its distributional solutions. Furthermore we choose to restrict attention to distributions of the form

$$
z(t)=\xi(t) H(t)+c_{0} \delta(t)+c_{1} \delta^{(1)}(t)+\cdots+c_{h} \delta^{(h)}(t),
$$

where $\xi(t)$ is an exponential polynomial, $H(t)$ is Heaviside's unit step function, $c_{k}$ is a constant and $\delta^{(k)}$ is the $k$-th derivative of the delta function. We can write

$$
z(t)=\xi(t) H(t)+p(D) \delta(t)
$$

where $p(s)=c_{0}+c_{1} s+\cdots+c_{h} s^{h}$ is a polynomial. We will call $z_{\infty}(t)=$ $p(D) \delta(t)$ the impulsive part of $z(t)$. Given two such distributions $z$ and $\tilde{z}$, we will say that $z$ is quasi-equal to $\tilde{z}$, and write $z \equiv \tilde{z}$, if $z$ and $\tilde{z}$ have the same impulsive part.

The distributional derivative of $z(t)$ has the same form, in fact

$$
D z(t)=(D \xi(t)) H(t)+[\xi(0)+D p(D)] \delta(t) .
$$

Convolution products also have the same form; in particular, for any complex number $\lambda$,

$$
e^{\lambda t} H(t) * p(D) \delta(t)=r e^{\lambda t} H(t)+q(D) \delta(t),
$$

where $q(s)=[p(s)-p(\lambda)] /(s-\lambda)$ is the quotient and $r=p(\lambda)$ the remainder in the division of $p(s)$ by $s-\lambda$. The Laplace transform of $z(t)$ is

$$
\hat{z}(s)=\hat{\xi}(s)+p(s) \text {, }
$$


where $\hat{\xi}(s)$ is the classical Laplace transform of $\xi(t)$. Moreover, for an arbitrary polynomial $q(s)$ the Laplace transform of $q(D) z(t)$ is $q(s) \hat{z}(s)$.

LEMMA 3. For any impulsive input $u(t)=q(D) \delta(t)$, where $q(s) \in \mathbf{C}^{m}[s]$, the polynomial system (9) has a unique distributional solution of the form

$$
x(t)=\xi(t) H(t)+p(D) \delta(t),
$$

where $p(s) \in \mathbf{C}^{n}[s]$. Moreover $\xi(t)$ is a solution of the homogeneous equation

$$
T(D) \xi=0 \text {. }
$$

Proof. Suppose we are given a solution of (9). If $x(t)=\xi(t) H(t)+p(D) \delta(t)$, then on taking Laplace transforms we obtain

$$
T(s) \hat{\xi}(s)+T(s) p(s)=U(s) q(s) .
$$

Thus $\xi(s)$ and $p(s)$ are the strictly causal and polynomial parts of the rational vector $T^{-1}(s) U(s) q(s)$. This shows that $x(t)$ is uniquely determined and, by Lemma 1 , that $\xi(t)$ is a solution of (1). Then $y(t)=V(D) x(t)+W(D) u(t)$ is also uniquely determined.

Conversely, we obtain a solution of (9) if we define $\hat{\xi}(s)$ and $p(s)$ in this way and take $\xi(t)$ to be the inverse Laplace transform of $\hat{\xi}(s)$.

In the situation of Lemma 3 we will say that the solution $\xi(t)$ of the homogeneous equation (1) is the regular part of the solution of (9) with the impulsive input $u(t)$.

With this terminology the results stated in the first part of Section 3 admit the following dynamical interpretation. By Lemma 1 , the state-space $\mathscr{X}$ is the set of Laplace transforms of all solutions of the equation

$$
Q(D)\left(\begin{array}{l}
x \\
u \\
y
\end{array}\right)=0
$$

that is,

$$
u=0, \quad T(D) x=0, \quad y=V(D) x .
$$

We recall also that controllability means that the map $\pi Q^{-1}(s) \mathscr{B}: \mathrm{C}^{m}[s] \rightarrow \mathscr{X}$ is surjective, and observability means that the map $\mathscr{C}: \mathscr{X} \rightarrow \pi \mathbf{C}^{p}(s)$ is injective. We conclude that the polynomial system (9) is controllable if every solution of the homogeneous equation (1) is the regular part of the solution of (9) for some impulsive input $u(t)$. It is observable if $\xi=0$ is the only solution of (1) for which $V(D) \xi=0$.

Furthermore, the polynomial system (9) is system equivalent to the polynomial system

$$
\tilde{T}(D) \tilde{x}=\tilde{U}(D) u, \quad y=\tilde{V}(D) \tilde{x}+\tilde{W}(D) u
$$


if the transfer matrices are the same, i.e.

$$
W(s)+V(s) T^{-1}(s) U(s)=\tilde{W}(s)+\tilde{V}(s) \tilde{T}^{-1}(s) \tilde{U}(s),
$$

and there exists a one-to-one linear map $\varphi: \xi(t) \rightarrow \tilde{\xi}(t)$ of all solutions of the homogeneous equation (1) onto all solutions of the homogeneous equation

$$
\tilde{T}(D) \tilde{\xi}=0
$$

such that

(i) $\varphi[D \xi(t)]=D[\varphi \xi(t)]$,

(ii) $V(D) \xi(t)=\tilde{V}(D)[\varphi \xi(t)]$,

(iii) if $\xi(t)$ is the regular part of the solution of (9) with some impulsive input $u(t)$, then $\varphi \xi(t)$ is the regular part of the solution of (10) with the same input.

Indeed (i), in conjunction with the linearity of $\varphi$, says that the map $f$ in Figure 2 is a homomorphism, while (ii) and (iii) say that the right and left halves of this diagram commute.

We consider next the corresponding results for behaviour at infinity. A distribution $(x, u, y)$ will be said to be a quasi-solution of the polynomial system (9) if

$$
Q(D)\left(\begin{array}{l}
x \\
u \\
y
\end{array}\right) \equiv 0,
$$

that is,

$$
u \equiv 0, \quad T(D) x \equiv U(D) u, \quad y \equiv V(D) x+W(D) u .
$$

The impulsive part $\left(x_{\infty}, 0, y_{\infty}\right)$ of a quasi-solution will be called an impulsive solution. We draw attention to the fact that in general $y_{\infty}$ is not uniquely determined by $x_{\infty}$.

If $(x, u, y)$ is a quasi-solution of $(9)$, then so also is the convolution product $\exp (\lambda t) H(t) *(x, u, y)$, for any complex number $\lambda$. It follows that if $\left(x_{\infty}, 0, y_{\infty}\right)$ is an impulsive solution of (9), then so also is the impulsive part $\Delta_{\lambda}\left(x_{\infty}, 0, y_{\infty}\right)$ of $\exp (\lambda t) H(t) *\left(x_{\infty}, 0, y_{\infty}\right)$.

By Lemma 2, the state-space at infinity $\mathscr{X}_{\infty}$ is the set of Laplace transforms of all impulsive solutions of (9). Hence the results of Section 2 admit the following dynamical interpretation. The polynomial system (9) is controllable at infinity if every impulsive solution of (9) is the impulsive part of a solution (and not merely of a quasi-solution). It is observable at infinity if it has no nontrivial impulsive solution $\left(x_{\infty}, 0, y_{\infty}\right)$ with $y_{\infty}=0$.

The polynomial system (9) is system equivalent at infinity to the polynomial system (10) if the transfer matrices are the same and there exists a one-to-one linear map $\psi:\left(x_{\infty}, 0, y_{\infty}\right) \rightarrow\left(\tilde{x}_{\infty}, 0, \tilde{y}_{\infty}\right)$ of all impulsive solutions of (9) onto all 
impulsive solutions of (10) such that

(i) $\psi\left[\Delta_{\lambda}\left(x_{\infty}, 0, y_{\infty}\right)\right]=\Delta_{\lambda}\left[\psi\left(x_{\infty}, 0, y_{\infty}\right)\right]$ for all $\lambda \in \mathbf{C}$,

(ii) $\tilde{y}_{\infty}=y_{\infty}$,

(iii) if $\left(x_{\infty}, 0, y_{\infty}\right)$ is the impulsive part of the solution of (9) with input $u(t)$, then $\psi\left(x_{\infty}, 0, y_{\infty}\right)$ is the impulsive part of the solution of (10) with the same input.

These results follow in the same way as the previous ones. The parallel between ordinary system equivalence and system equivalence at infinity could have been made more complete, but this would have concealed the simplifications which are present in the ordinary case.

\section{References}

[1] B. D. O. Anderson, W. A. Coppel and D. J. Cullen, "Strong system equivalence (I)", J. A ustral. Math. Soc. Ser. B 27 (1985), 194-222.

[2] W. A. Coppel, "Some remarks on strict system equivalence", Proc. 5th Internat. Symp. Math. Theory of Networks and Systems, Santa Monica (1981), 33-34.

[3] D. Hinrichsen and D. Prätzel-Wolters, "Solution modules and system equivalence", Internat. J. Control 32 (1980), 777-802.

[4] G. C. Verghese, "Infinite-frequency behavior in generalized dynamical systems", Ph. D. Dissertation, Stanford University, 1978. 